\title{
A New Image Quality Assessment Algorithm based on SSIM and Multiple Regressions
}

\author{
Zhengyou Wang ${ }^{1,}$, Liying $\mathrm{Li}^{1}$, Shuang $\mathrm{Wu}^{2}$, Yanhui Xia ${ }^{1}$, \\ Zheng Wan ${ }^{2}$ and Cong Cai ${ }^{1}$ \\ ${ }^{1}$ Shijiazhuang Tiedao University; ${ }^{2}$, Jiangxi University of Finance \& Economics \\ ${ }^{1}$ No. 17 Northeast, Second Inner Ring; ${ }^{2}$ No.169, East Shuanggang Road \\ ${ }^{1}$ Shijiazhuang, Hebei, P.R.C. 050043; ${ }^{2}$ Changbei, Nanchang, Jiangxi, P.R.C. \\ 330013 \\ *zhengyouwang@stdu.edu.cn,lly_0803@126.com,w.s.617@163.com, \\ yanhuixia@stdu.edu.cn,cloudcity66@163.com,cai_cong@126.com
}

\begin{abstract}
Image quality assessment (IQA) is crucial in image processing algorithms. In the state-of-the-art IQA index, the structural similarity (SSIM) index has been proved to be better objective quality assessment metric. However, the accuracy of SSIM is relatively lacking when used to access blurred images. And the component weights of structural similarity (SSIM) index are fixed in some past environments. So an improved assessment algorithm incorporating multiple linear regressions and SSIM index was proposed in this paper. We use regression analysis to adjust the component weight of SSIM index. So the improved algorithm is more accuracy on different distortion types' quality assessment. Experimental results show that the improved SSIM algorithm is better than traditional methods in nonlinear regression correlation coefficient, Spearman correlation coefficient and out ratio.
\end{abstract}

Keywords: image quality assessment; SSIM; multiple linear regressions; gradient

\section{Introduction}

The assessment of image quality is important in numerous image processing applications. According to gained information, quality evaluation methods can be divided into full-reference quality assessment, no-reference quality assessment and reducedreference quality assessment [1]. In the full-reference quality evaluation, the most classic models are peak signal-to-noise ratio (PSNR) and mean square error (MSE). Such methods are simple arithmetic and the physical meaning is clear. However, these methods are based on pixel differences and do not take into account the spatial structure and human visual characteristics. Natural images have a specific structure and a strong interpixel dependent relationship that reflects the structure of the visual information in the scene. Based on this point, Wang et. al., proposed structure similarity algorithm (SSIM) [1], considering three components of image brightness, contrast and structural similarity from the perspective of spatial structure. Reference [2] analyzed the understanding of the mathematical properties of the SSIM measure. There are some other improved methods. Reference [3] considered an RR-IQA method by estimating the structural similarity index (SSIM), Due to the performance of SSIM is less effective when used to rate blurred and noisy images,[4] proposed a four-component image model that classifies image local regions according to edge and smoothness properties.

* Corresponding Author 
This paper aims at objective evaluation methods reflecting the inherent nature of images and closing to human subjective feelings. SSIM index bypassed natural images' content and multi-channel complex problems in some extent, to assess structure of the original image and image to be evaluated directly. The evaluation result of the original SSIM algorithm for fuzzy type of distortion is not accurate enough. So we introduced gradient information to SSIM index. On the basis, this paper proposed an improved SSIM index by using regression analysis to adjust the component weight of original SSIM index. So we can get the prediction model for different image distortion types. In addition, we consider a united model for all image distortion types when distortion type is unknown. Experimental results show that improved index has a better correlation with subjective evaluation.

The rest of this paper is organized as follows. Section 2 proposed an improved image quality assessment algorithm based on gradient and SSIM. On the basis of Section 2, A SSIM index integrated with multiple regressions is discussed in Section 3. In Section 4, we analyze and explain experimental results. And a general conclusion is presented in Section 5 .

\section{Improved Image Quality Assessment Algorithm Based on Gradient and Structural Similarity}

\subsection{Gradient Component}

Contents of the image can generally be classified as edge, texture, and flat regions. According to the human visual characteristics, human eyes are most sensitive to edges and texture information of images. Image gradient may reflect the changes of edges and texture well. So it can be used to evaluate the clarity of images. This article only needs magnitude of the gradient to reflect the gray changes, and we do not require extract image edges strictly. Sobel operators are used here to calculate the image gradient, as shown in Figure 1. The left is horizontal edge detection operator $x$. The right is vertical edge detection operator.

$$
\left[\begin{array}{ccc}
-1 & -2 & -1 \\
0 & 0 & 0 \\
1 & 2 & 1
\end{array}\right] \quad\left[\begin{array}{lll}
-1 & 0 & 1 \\
-2 & 0 & 2 \\
-1 & 0 & 1
\end{array}\right]
$$

\section{Figure 1. Sobel Operators}

The pixels' gradient of image coordinates $(i, j)$ is calculated by (1) approximately.

$$
G(i, j)=\left|d x_{i, j}\right|+\left|d y_{i, j}\right|
$$

We use the horizontal and vertical edge operator to each pixel in the image for differential operational. Operation process is as (2):

$S_{-} h(x, y)=F(x-1, y+1)+2 F(x, y+1)+F(x+1, y+1)-F(x-1, y-1)-2 F(x, y-1)-F(x+1, j-1)$

In Figure 2, the gradient of all the pixels from the image composed a "gradient" map. 


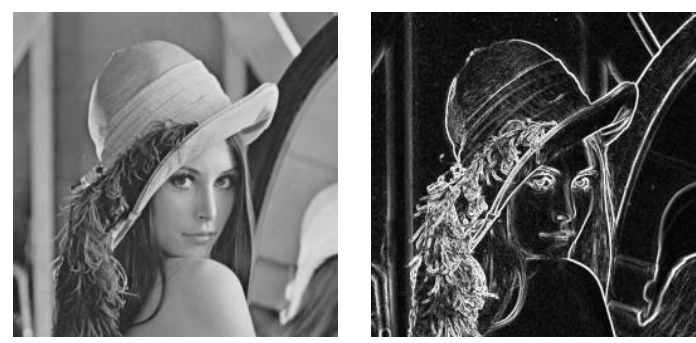

Figure 2. Lena and its "Gradient" Map

The "gradient" map of Lena is calculated by using the Sobel operators. We can see that it reflects edge and texture information of the original image accurately. If its luminance is brighter, the variation of pixel values is larger, corresponding to edge and texture information of the image. And if its luminance is darker, the variation of pixel values is smaller, corresponding to the flat area.

Luminance component of SSIM is used mean brightness of pixels in the image to define, and here gradient information will be added to the SSIM. So we can use a similar definition, there are:

$$
g(x, y)=\frac{2 \sum_{i} \sum_{j} G_{x}(i, j) G_{y}(i, j)+C}{\sum_{i} \sum_{j}\left[G_{x}(i, j)\right]^{2}+\sum_{i} \sum_{j}\left[G_{y}(i, j)\right]^{2}+C}
$$

$g(x, y)$ can be referred to the gradient similarity of the original image and the distortion one. In (3), $G_{x}(i, j), G_{y}(i, j)$ are gradient of pixels $(i, j)$ in the original image and the distortion one respectively. When calculated the gradient of the flat region, the numerator and denominator may appear zero, and therefore the numerator and denominator are added with a small constant value $\mathrm{C}$.

\subsection{Improved Image Quality Assessment Model Based on Gradient and Structural Similarity}

Gradient component is proposed mainly for fuzzy distortion. It can be seen as a factor reflecting structural differences of images, and further improving the SSIM algorithms. Therefore, new structure similarity evaluation equation is as follows:

$\operatorname{GSSIM}(x, y)=[l(x, y)]^{\alpha} \cdot[c(x, y)]^{\beta} \cdot[s(x, y)]^{\gamma} \cdot[g(x, y)]^{\lambda}$

Wherein $\alpha, \beta, \gamma, \lambda$ are used to adjust the weight of each component. Usually in the actual application, firstly, the reference image and distortion image are divided into blocks (e.g., of $8 \times 8$ ways). Then GSSIM value is calculated for each block and compute the average GSSIM value of the whole image. Therefore we can get the evaluation value of the entire image:

$\operatorname{MGSSIM} \quad(X, Y)=\frac{1}{M} \sum_{i=1}^{M} \operatorname{GSSIM}\left(x_{i}, y_{i}\right)$

$\mathrm{M}$ is the number of image sub-blocks. This block calculation more emphasis on the similarity of image details than calculate GSSIM value of the whole image, and more in line with human visual characteristics. Equation (5) means that the contribution of each block to evaluation of the whole image is the same, and in fact, human eyes have different interest in different regions. For objective evaluation more accurately, we have given different weights for different regions of images. So we can find the proportion ${ }^{w_{i}}$ that these blocks of original image account for the whole image, and make it as the weight of each block: 
$w_{i}=D_{i} / \sum_{i=1}^{M} D_{i}$

$D_{i}$ is the variance of each sub-block. Local variance of images contains a large amount of structural information, which reflects intensity of local pixels' changes. If the variance of block is large, indicating that the area of pixels' value is fluctuating. It means that there is an edge or texture and it is the region that human visual system is interested. Then the weight of this image block is larger; vice versa. So assessment formula based on the weighted content and SSIM is as follows:

$$
\operatorname{WGSSIM} \quad(X, Y)=\sum_{i=1}^{M} w_{i} \operatorname{GSSIM} \quad\left(x_{i}, y_{i}\right)
$$

\section{Structural Similarity Index Method Integrated with Multiple Regression of Image Quality Assessment}

\subsection{Multiple Regression Analysis Theory}

Multivariate statistical analysis [5] studies statistical regularity of interdependence among multiple variables in objective things. Regression analysis is one of the most common of it. According to how much regression analysis involving independent variables, it can be divided into a regression analysis and multiple regression analysis; According to the types of relationship between independent variables and dependent variable, regression analysis can be divided into linear regression and nonlinear regression analysis. If the regression analysis includes only one independent variable and a dependent variable, and the relationship between the two can be used in a straight line approximately, this regression analysis is called a linear regression analysis. If the regression analysis includes two or more independent variables, and the relationship between the dependent and independent variables is linear, it is called multiple linear regression analysis. In this paper, we mainly use multiple linear regression equation for regression analysis.

Regression analysis is an effective tool by establishing statistical model that studies closeness, structural state, model prediction of variables each other inter-relationship. The process of establishing regression model of practical problems is as follows:

(1) According to the research purpose, set indicator variables. Regression analysis model is mainly revealing the number of contacts between relevant variables of things. Set dependent variable y according to the purpose of the problem, and then select some variables which have statistical relationship with y as independent variables. Use relevant theories, from a qualitative point of view of the study to determine the causal relationship between the factors in question.

(2) Collect and collate statistics. The establishment of model is based on sample statistics of regression variables. When the regression model of variables is determined, we must collect, collate data for these variables.

(3) Determine the mathematical form of the theoretical regression model. We sometimes can not determine the form of model according to information obtained, and then we can use different forms of computer simulation. For the different simulation results, choose a better choice as a theoretical model.

(4) Estimate parameters of model. The main part of regression analysis is to use the collated sample data to estimate parameters of the model. The most commonly used method is the least squares method, which is a classic estimation method. 
(5) Tests and modification of the model. When the unknown parameters of the model are estimated, a regression model is initially established. It is clearly not enough careful to use this model to predict, control and analysis immediately. Statistical tests must be carried out on the model. Statistical test is usually a regression equation significance test, and significance test of regression coefficients, as well as the goodness of fit test and so on. The tests includes t test, F test and good.

(6) The application of regression model. When the regression model through a variety of statistical tests and models have a reasonable sense, we can use this model to further study similar problems.

\subsection{Structural Similarity Integrated with Multiple Regression Analysis of Image Evaluation Model}

The improved SSIM index proposed in Section 2 added gradient information to the SSIM evaluation algorithm. The improved SSIM evaluation equation is (4).

In past experiments of SSIM algorithm, for the purpose to simplify the experiment, evaluation formula weights of all the components $(\alpha, \beta, \gamma, \lambda)$ are taken to 1 generally, which ignores the different importance of each component and different characteristics of distortion types. This paper is to study contribution of each component to obtain the final evaluation results, therefore how to distribute weights of each component for different types of distortion is the key.

Equation (4) shows that the independent variables are determined, i.e., the two images luminance signal $l(x, y)$, contrast information $c(x, y)$, the structure information $s(x, y)$ and the gradient information $g(x, y)$. Regression model was designed to predict the quality of the image, so the subjective evaluation values are made as the dependent variable to maintain consistency with the subjective perception. In (4), independent variables and the dependent variables are non-linear relationship. But it can be converted to a linear relationship-taking (4) the logarithm are:

$\ln [\operatorname{GSSIM}(x, y)]=\alpha \ln [l(x, y)] \bullet \beta \ln [c(x, y)] \bullet \gamma \ln [s(x, y)] \bullet \lambda \ln [g(x, y)]$

The above equation is simplified to:

$y=\alpha x_{1}+\beta x_{2}+\gamma x_{3}+\lambda x_{4}$

Thus we can use multiple linear regression models to explain (8). LIVE image database [6] is used to conduct experiments. We use each image to create a regression equation, and the regression equation must satisfy assumptions. Use least squares regression [7] equations to estimates model parameters and related hypothesis testing will get the results in the experiment. Specific algorithm steps are as follows:

(1) Establish model components. Model components are determined i.e., the component of luminance, contrast, structural and gradient. We conduct multiple linear regression analysis on LIVE image database, calculate parameters $(\alpha, \beta, \gamma, \lambda)$ via least squares method. So that we can obtain models to evaluate the image quality;

(2) The obtained model must be carried out by F-test, t test and goodness of fit test; if one does not pass the test, the model will have to be modified;

(3) The examined model can be used to evaluate the quality of distorted images;

(4) When the type of image distortion is known, select the corresponding distortion model to evaluate the image quality; Use a generic model to evaluate the image quality when distortion type is unknown. 


\section{Experimental Results and Analysis}

Simulation experiments are on the LIVE image database, a total of 779 distorted image: JPEG2000 (169 images), JPEG (175 images), WN (145 images), GBLUR (145 images) and FF (145 images). Meanwhile the database gives DMOS (Difference Mean Opinion Scores) of each distortion images 。 Measure $l(x, y), c(x, y), s(x, y)$ and $g(x, y)$ of all the distortion images with respect to the reference image Due to $l(x, y)=1$ in all measured image, so the parameters to be adjusted include $\beta, \gamma, \lambda$.

When the type of distortion is determined, we make linear regression analysis for each class to obtain the corresponding model parameters. Then examine the model with goodness of fit, $\mathrm{F}$ test and $\mathrm{t}$ test. Table 1 is weight coefficients obtained respectively and the corresponding test. The measured $l(x, y)=1$, so we can ignore it. As can be seen from the table, after linear regression, goodness of fit $R^{2}$ of each type of distortion model is higher. R shall be correlation coefficient of the variable with all independent variables; For the F-test, look up F distribution tables, and we can find that $\mathrm{F}$ values are far greater than the critical value. So reject original hypothesis $H_{0}$, and believe $y$ for $x_{1}, x_{2}, \ldots, x_{p}$ is significant linear relationship in the significance level $=0.05$.Then the regression equation is significant. For $\mathrm{t}$ test, look up t distribution table to find the critical value $t_{\alpha / 2}$ of two-sided test, where t-values are in line with $\left|t_{j}\right| \geq t_{\alpha / 2}$ and the corresponding P values are very small, indicating $x_{1}, x_{2}, \ldots, x_{p}$ of each independent variable for $\mathrm{y}$ is significant linear relationships.

In fact, in many cases when the images are evaluated, we can not make sure what kind of image distortion exists. So conduct linear regression for all types of image distortion to get a general model. Even in the case of image distortion type is unknown; this model can be used to predict the quality. Table 2 is a unified forecasting model and its test after linear regression. Linear relationship between independent variables and the dependent variable is significant from F-test and t-test we can see. But the value of goodness of fit $R^{2}$ is small and it seems that the effect is not very good, which is due to the model includes all types of image distortion. But ultimately determine whether this general model is good depends on consistency with subjective evaluation value.

In this paper, we use three quantitative indicators to evaluate the algorithm:

(1) CC: the correlation of objective and subjective scores after nonlinear regression analysis, which measures the accuracy of the predicted model.

(2) SROCC: Spearman correlation coefficient between objective and subjective scores, which detects the monotony of algorithms.

(3) OR (outline-ratio): the proportion of points in two times larger than standard deviation after non-linear fitting prediction, which measures the stability.

Table 1. Regression Model of All Types of Distortion and Test

\begin{tabular}{|c|c|c|c|c|c|c|}
\hline Distortion types & Component & Coefficients & $R^{2}$ & F values & $\mathrm{t}$ values & $\begin{array}{c}\text { Corresponding } \mathrm{P} \\
\text { value }\end{array}$ \\
\hline JPEG2000 & \multirow{5}{*}{$\begin{array}{l}c(x, y) \\
s(x, y) \\
g(x, y)\end{array}$} & $\begin{array}{c}-200.1371 \\
14.3311 \\
2.0180 \\
\end{array}$ & 0.8410 & 290.8682 & $\begin{array}{c}-8.8724 \\
9.3679 \\
11.5347 \\
\end{array}$ & $\begin{array}{c}1.1393 \mathrm{e}-15 \\
5.4561 \mathrm{e}-17 \\
6.0 \mathrm{e}-23 \\
\end{array}$ \\
\hline JPEG & & $\begin{array}{c}-877.0913 \\
11.8575 \\
4.9189 \\
\end{array}$ & 0.7888 & 206.6394 & $\begin{array}{c}-3.3715 \\
4.8429 \\
10.9909 \\
\end{array}$ & $\begin{array}{c}0.0009 \\
2.9135 \mathrm{e}-06 \\
0 \\
\end{array}$ \\
\hline GBLUR & & $\begin{array}{c}-19.9665 \\
3.8789 \\
0.7831 \\
\end{array}$ & 0.9481 & 859.2707 & $\begin{array}{c}-6.7612 \\
5.0412 \\
19.5699 \\
\end{array}$ & $\begin{array}{c}3.3519 \mathrm{e}-10 \\
1.397 \mathrm{e}-06 \\
5.0321 \mathrm{e}-42 \\
\end{array}$ \\
\hline \multirow[t]{2}{*}{ WN } & & $\begin{array}{c}-8.8476 \\
1.5081 \\
1.9381 \\
\end{array}$ & 0.8368 & 498.4093 & $\begin{array}{c}-3.7772 \\
11.0006 \\
7.4758 \\
\end{array}$ & $\begin{array}{c}0.0002 \\
1.037 \mathrm{e}-11\end{array}$ \\
\hline & & $\begin{array}{c}-0.5438 \\
1.5993 \\
1.5352 \\
\end{array}$ & 0.8192 & 435.4041 & $\begin{array}{c}3.6700 \\
-2.5009 \\
15.3279 \\
\end{array}$ & $\begin{array}{c}0.0004 \\
0.0136 \\
1.653 \mathrm{e}-31 \\
\end{array}$ \\
\hline
\end{tabular}


Table 2. United Model for All Distortions and Test

\begin{tabular}{c|c|c|c|c|c}
\hline Unified model & Coefficients & $R^{2}$ & F value & T value & $\begin{array}{c}\text { corresponding } \\
\text { P value }\end{array}$ \\
\hline$l(x, y)=1$ & -18.6041 & & & -5.2779 & $1.7104 \mathrm{e}-07$ \\
$c(x, y)$ & 2.5892 & 0.5605 & 658.5166 & 11.7224 & 0 \\
$s(x, y)$ & 1.6929 & & & 19.3335 & 0 \\
$g(x, y)$ & & & \\
\hline
\end{tabular}

Table 3. Experiment Results on LIVE Database

\begin{tabular}{c|c|c|c|c}
\hline Algorithm & Tested images & CC & SROCC & OR \\
\hline \multirow{4}{*}{ SSIM } & JPEG2000 & 0.9692 & 0.9614 & 0.4897 \\
\cline { 2 - 5 } & JPEG & 0.9808 & 0.9764 & 0.3931 \\
\cline { 2 - 5 } & GBLUE & 0.9274 & 0.9216 & 0.5379 \\
\cline { 2 - 5 } & WN & 0.9775 & 0.9694 & 0.4828 \\
\cline { 2 - 5 } & FF & 0.9515 & 0.9556 & 0.5448 \\
\cline { 2 - 5 } & JPEG2000 & 0.9763 & 0.9646 & 0.4803 \\
\cline { 2 - 5 } & JPEG & 0.9877 & 0.9725 & 0.4621 \\
\cline { 2 - 5 } & GBLUR & 0.9727 & 0.9681 & 0.4207 \\
\cline { 2 - 5 } WGSSIM & WN & 0.9787 & 0.9734 & 0.4552 \\
\hline \multirow{4}{*}{\begin{tabular}{c} 
model \\
\cline { 2 - 5 }
\end{tabular}} & JPEG2000 & 0.9769 & 0.9653 & 0.3311 \\
\cline { 2 - 5 } & JPEG & 0.9770 & 0.9664 & 0.4789 \\
\cline { 2 - 5 } & GBLUR & 0.9728 & 0.9774 & 0.3529 \\
\cline { 2 - 5 } & WN & 0.9778 & 0.9659 & 0.3724 \\
\hline \multirow{4}{*}{$\begin{array}{c}\text { United } \\
\text { regression } \\
\text { model }\end{array}$} & JPF & 0.9728 & 0.9643 & 0.4286 \\
\cline { 2 - 5 } & JPEG & 0.9752 & 0.9552 & 0.3262 \\
\cline { 2 - 5 } & GBLUR & 0.9786 & 0.9733 & 0.3176 \\
\cline { 2 - 5 } & WN & 0.9785 & 0.9650 & 0.3931 \\
\hline
\end{tabular}

Table 3 is algorithm performance comparison on LIVE image database. WGSSIM, regression model, general regression model are superior to the SSIM from correlation coefficients, and have a better correlation with subjective evaluation. The out ratio of regression model and general regression model are lower compared with others, indicating that the image quality predictions are more accurate.

Figures below are scatters of SSIM index and regression model in sub-database of LIVE. 

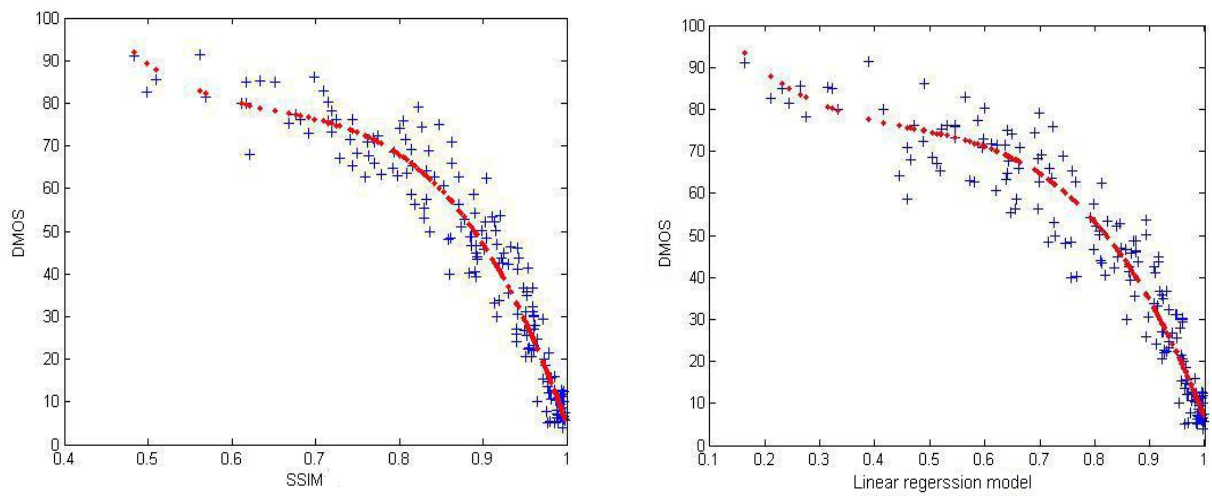

Figure 3. The Scatter Diagram of Results on JPEG2000 Image Database
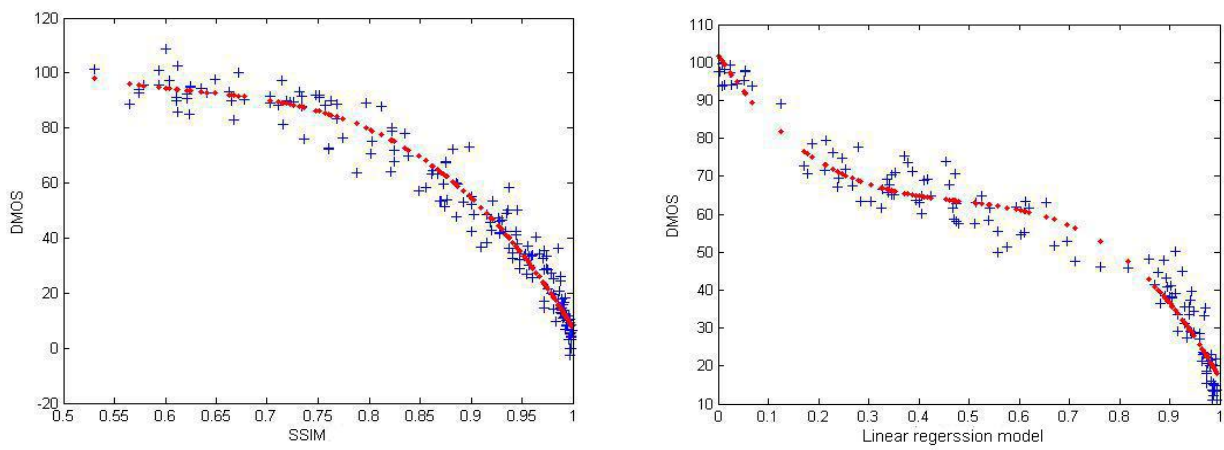

Figure 4. The Scatter Diagram of Results on JPEG Image Database
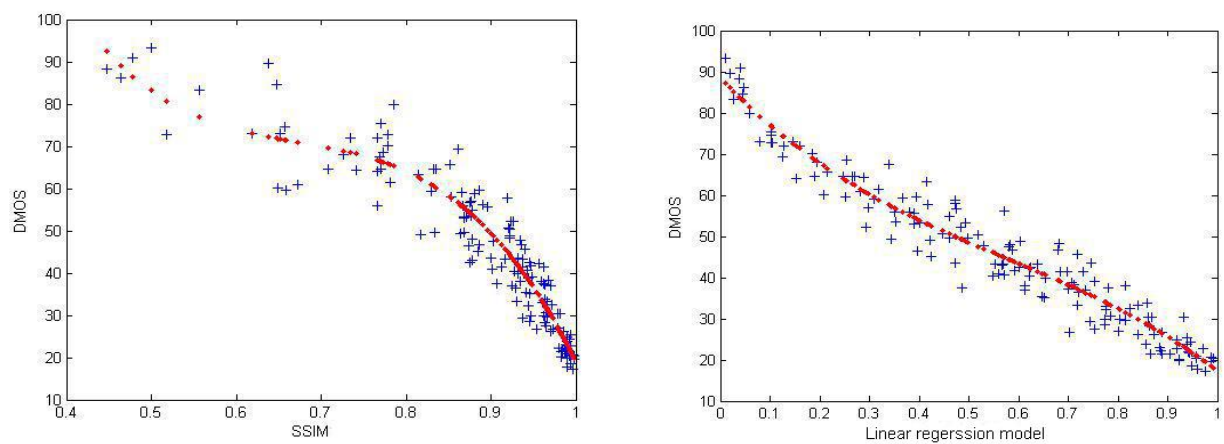

Figure 5. The Scatter Diagram of Results on GBLUR Image Database
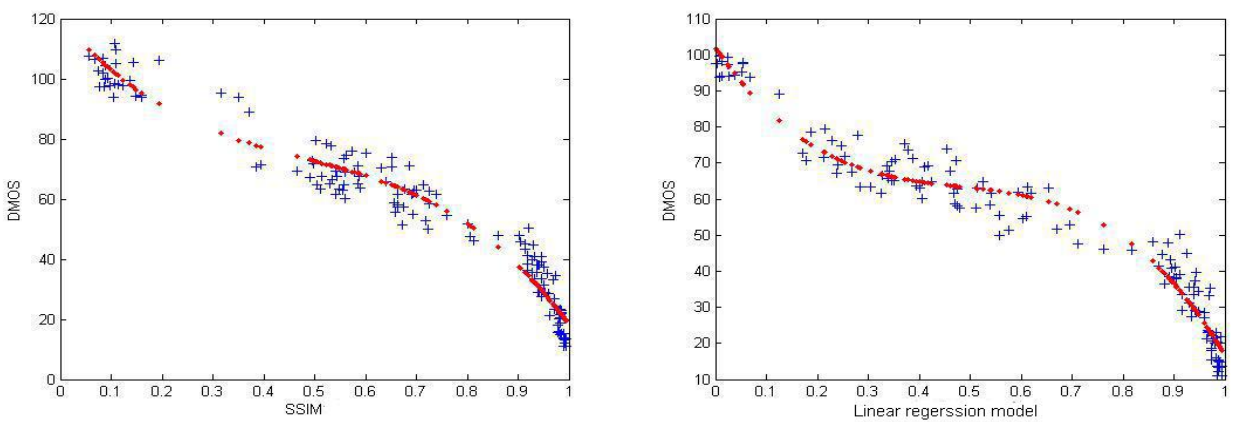

Figure 6. The Scatter Diagram of Results on Gauss White Noise Image Database 

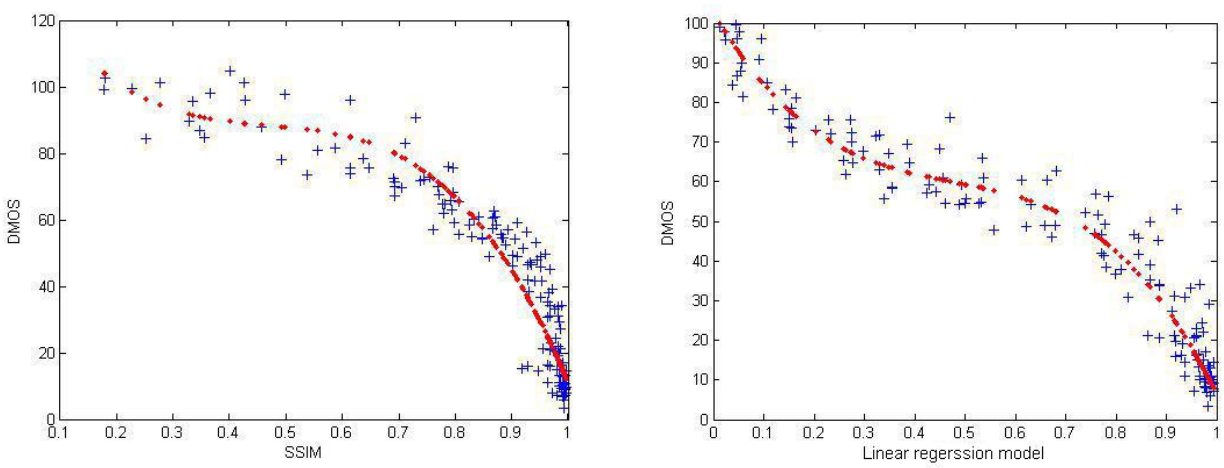

Figure 7. The Scatter Diagram of Results on FAST FADING Image

\section{Conclusion}

In this paper, to solve the problem that SSIM index is not accurate for fuzzy distortion, we added gradient information to the SSIM algorithm as a new component. An algorithm based on gradient and structural similarity was proposed. Based on this, multiple linear regression analysis was used to adjust weights of each component, and then use the gained model to evaluate the image quality. Adjusting the weight of the component can reflect relative importance degrees of each component and the difference between diverse types of distortion. Various distortion types have different evaluation models. There are general evaluation model of all types. General model is relatively easy to use. When the type of distortion in the image is not clear; a common evaluation model is used to predict its quality. Experiments show that the improved SSIM index is closer to subjective perception.

\section{Acknowledgments}

This work was partially supported by the National Natural Science Foundation of China (No: 60963011, 61162009), the Jiangxi Natural Science Foundation of China (No: 2009GZS0022).

\section{References}

[1] Z. Wang, A.C. Bovik, H. R. Sheikh and E. P. Simoncelli, "Image quality assessment: From error visibility to structural similarity”, Image Processing IEEE Transactions on, vol. 13, no. 4, (2004), pp. 600-612.

[2] Brunet, Dominique, R. Edward and Z. Wang, "On the mathematical properties of the structural similarity index”, Image Processing, IEEE Transactions on, vol. 21, no. 4, (2012), pp. 1488-1499.

[3] A. Rehman and Z. Wang, "Reduced-Reference Image Quality Assessment by Structural Similarity Estimation", Image Processing, IEEE Transactions on, vol. 21, no. 8, (2012), pp. 3378-3389.

[4] C. Li and A. C. Bovik, "Content-partitioned structural similarity index for image quality assessment”, Signal Processing: Image Communication, vol. 25, no. 7, (2010), pp. 517-526.

[5] A. S. Chatterjee Hadi, "Regression analysis by example", John Wiley \& Sons, (2013).

[6] H. R. Sheikh, Z.Wang and L. Cormack, "LIVE image quality assessment database release 2 [DB / OL]. http://live.ece.utexas.edu / research / quality, (2011).

[7] Z. Wang, W. Wang, Z .Wan and Y. Xia, "No-reference hybrid video quality assessment based on partial least squares regression", Multimedia Tools and Applications, DOI: 10.1007/s11042-014-2166-0, no. 7, (2014), pp. 1-14. 
International Journal of Signal Processing, Image Processing and Pattern Recognition Vol.8, No.11 (2015) 